\title{
Syncytial nuclear aggregates as markers for villous maturation in placentas from preterm birth
}

\author{
Madita Reimer \\ Department of Anatomy and Embryology \\ ma.reimer@student.maastrichtuniversity.nl
}

\section{Abstract}

The syncytiotrophoblast is the multinucleated syncytial layer lining the placental villi. In some areas nuclei form aggregates termed true knots by the process of trophoblast turnover. Thereby, the underlying cytotrophoblast cells proliferate, differentiate and fuse with the overlying synctiotrophoblast cells which have no own generative potency. The nuclei in the syncytiotrophoblast are undergoing apoptosis and form true knots which are shed into the maternal circulation as syncytiotrophoblast microparticles (STBMs). This process ensures that the diffusion distance between maternal and foetal blood is decreased to nourish the growing foetus. Excessive formation of true knots has been reported in placentas of pregnancies complicated by pre-eclampsia. This is a complication of pregnancy characterized by increased maternal blood pressure and induced preterm labour is the only treatment. Pregnancies complicated by chorioamnionitis, a severe inflammation of the foetal membranes due to a bacterial infection, are also at increased risk of preterm labour.

The aim of this study is to establish an objective scoring system for placental villous maturation based on true knots, STBMs and syncytial knotting frequency. Our assumption is that the frequency of syncytial knots in placentas complicated by pre-eclampsia are increased. True knots, STBMs and syncytial knotting are assessed by histological examination of placental tissue using a light microscope.

In pre-eclamptic placentas the highest numbers of true knots, syncytial knotting and STBMs compared to an idiopathic preterm control group were found. Placentas complicated by chorioamnionitis show a slight decrease of true knots, syncytial knotting and STBMs compared to the idiopathic preterm group and a significant decrease compared to pre-eclamptic placentas. The increase of true knots and syncytial knotting could be a 
sign for an increased trophoblast turnover during pre-eclampsia. This implies enhanced proliferation, differentiation and apoptosis of the placental epithelium. Chorioamnionitis placentas display lower number of true knots indicating a lower trophoblast turnover which may be part of another anomaly of the placental development.

For our scoring system are true knots, STBMs and syncytial knotting convincing markers for analysis of villous maturation in pre-eclampsia. In case of chorioamnionitis other markers may be more useful.

\section{Introduction}

The human placenta is the crucial connection between the maternal and foetal circulation. At term, it is approximately $15-20 \mathrm{~cm}$ in diameter, in the centre $3 \mathrm{~cm}$ thick and with a typical weight around $500 \mathrm{~g}(1)$. This organ consists macroscopically of the chorionic plate that attaches to the umbilical cord and the basal plate facing the maternal side. Foetal oxygen supply and carbon dioxide excretion is provided by gas exchange within the placenta. This gas exchange between maternal and foetal circulation takes place at the villous trees. The space in between the villi is filled with maternal blood called intervillous space (2). The placental barrier consists of syncytiotrophoblast, basal lamina of the foetal capillary, capillary endothelium and in early pregnancy also of cytotrophoblast in order to inhibit mixing of blood $(1,3)$.

The chorionic villi in the human placenta are covered by villous trophoblast which consists of cytotrophoblast and syncytiotrophoblast. The syncytiotrophoblast layer in contact with maternal blood consists of multinucleated syncytium and shows transcriptional activity (4). The underlying cytotrophoblast layer proliferates and differentiates and fuses with the overlying syncytiotrophoblast layer by the process of syncytial fusion (Figure 1). This maintains the growth of syncytiotrophoblast and incorporates new nuclei and organelles because it has no generative potency. After 3-4 weeks the nuclei in the syncytiotrophoblast become apoptotic and are shed into the intervillous space. This process called trophoblast turnover expands the syncytium in surface area and increases the efficiency of oxygen and nutrient exchange to provide the growing foetus with sufficient oxygen. Trophoblast turnover increases with gestational age and is more frequent in mature placentas. As a result it can be measured as a marker for villous maturation. Syncytiotrophoblast nuclei are sequestered together and form aggregates known as true syncytial knots to counterbalance the continuous input of material via syncytial fusion (5). They form protrusions of the apical membrane and are shed into the intervillous space as membrane- 
sealed structures that reach the maternal circulation without degeneration (Figure 1). These shed microparticles called syncytiotrophoblast microparticles (STBMs) can be detected in the maternal circulation (5).

In all mature placentas true syncytial knots can be found on many terminal villi. They are uncommon before 32 weeks of gestation and are increasing in frequency until term (6) and are used in placental examination to evaluate villous maturity. At term they are present at $10-30 \%$ of terminal villi (7). An excessive increase of syncytial knots has been reported in post-mature placentas (8). In addition, accumulation of nuclei showing no sign of chromatin condensation can be found. These structures were firstly described by Tenney and Parker in 1940 in pre-eclamptic placentas (9). Nowadays it is known that these structures are predominantly present during the third trimester of pregnancy, comprising inter-villous syncytial bridges and nuclear aggregates resulting from tangential flat sectioning across the syncytiotrophoblastic villous surface (10). Inter-villous syncytial bridges are formed by adjoining syncytial sprouts from adjacent chorionic villi (Figure 5). Hereafter, inter-villous bridges and nuclear aggregates due to tangential sectioned villi are referred as syncytial knotting according to Castellucci and Kaufmann (2). Syncytial knotting as a result of tangential flat sectioning is of interest because it can give information on the branching pattern of villi which may be altered in placental pathologies (2). The villous trophoblast turnover is altered in pregnancies complicated by pre-eclampsia.

Examination of placentas affected by pregnancy complications is accomplished by pathologists using standard protocol but it remains a subjective grading $(11,12)$. Moreover, foetal age is difficult to determine by histological examination between 28 and 36 weeks of gestation. An accurate histopathological assessment of placental villous maturation is important to identify causes of preterm delivery (11).

Therefore the primary purpose of this study was to establish a scoring system for villous maturation and trophoblast turnover based on true knots and syncytial knotting frequency. Previous studies have shown that placentas complicated by pre-eclampsia show higher maturity patterns than placentas derived from idiopathic preterm labour with the same gestational age $(13,14)$. Villous maturation in pre-eclampsia has been used as marker for defective placentation and placental vascular insufficiency (15). Consequently, we can use our preeclampsia data as internal validation for our scoring system. The same scoring system is transferred to another placental pathology by including a study group of placentas complicated by chorioamnionitis. 
We will analyse if there is also villous hypermaturation in chorioamnionitis placentas. The establishment of the maturity scoring was done by histological examination of paraffin embedded placentas. The frequency of true knots and syncytial knotting was analysed in an idiopathic preterm group, placentas complicated by pre-eclampsia or chorioamnionitis and a term group.

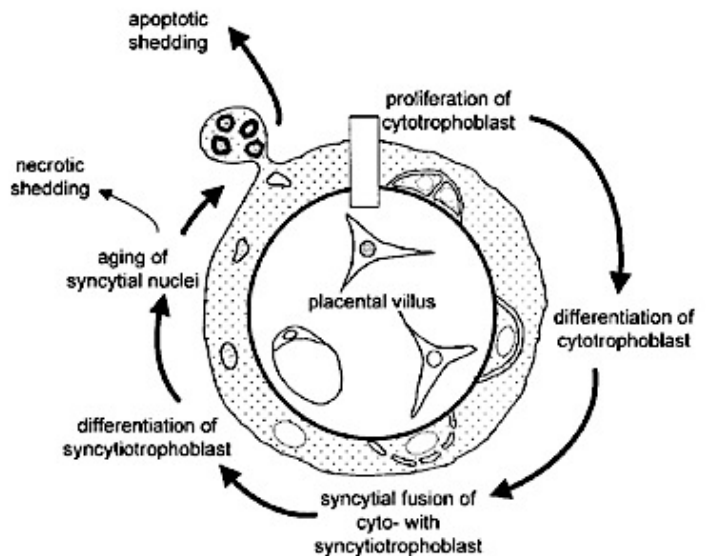

Figure 1. Villous trophoblast turnover. Schematic representation showing the villous trophoblast turnover in a clock-wise manner. The cytotrophoblast undergoes proliferation, differentiation and syncytial fusion with the syncytiotrophoblast. After the fusion the cellular content remains in the syncytiotrophoblast for 3-4 weeks. It undergoes differentiation and the aged nuclei are shed into the maternal circulation as syncytial knots. (adapted from (16))

\section{Material and methods}

\section{Patient Sampling}

The use of placental specimens was approved by the local Medical Ethical Committee of the Maxima Medical Centre for development Veldhoven, The Netherlands. Different placentas from pregnancies complicated by pre-eclampsia and chorioamnionitis were collected. Pregnant women, who delivered between January 2009 and December 2010 at a gestational age of 25 - 32 weeks, were eligible for the study. To compare placentas derived from normal pregnancies and abnormal pregnancies also placentas from women with idiopathic pre-term pregnancies were collected. After histological exclusion, 24 placentas from the idiopathic preterm group, 35 pre-eclamptic placentas and 41 placentas complicated by chorioamnionitis were included for further analysis. The placentas from six uncomplicated term pregnancies were used as control group and were collected at 
the Academic Hospital Maastricht after informed parental consent. Placental tissue from uncomplicated term pregnancies was included if birth was given at gestational age of at least 38.0 weeks and if no maternal or perinatal mortalities occurred during pregnancy.

\section{Immunohistochemistry}

The paraffin embedded placental sections were deparaffinised in xylene and hydrated by an ethanol series of $100 \%-50 \%$. Antigen retrieval was carried out by exposure to microwave treatment at 850 watt to boil with $10 \mathrm{mM}$ sodium citrate buffer $(\mathrm{pH} \mathrm{6.0)}$ to retrieve epitopebinding sites. After boiling the microwave was set at 80 watt for 5 minutes. Following cooling down at room temperature the sections were washed in demineralized water and PBS. Slides were further treated with $0.3 \%$ hydrogen peroxide in methanol for 30 minutes to block endogenous peroxidase activity. Non-specific binding was blocked with 30 minutes incubation in $5 \%$ normal goat serum diluted in PBS-Tween. The placental sections were incubated overnight at $4{ }^{\circ} \mathrm{C}$ with monoclonal mouse anti-human antibody against CD31 (clone JC7OA, Dako Denmark) (1:100) to stain endothelial cells. Sections were probed with biotin conjugated goat anti-mouse secondary antibody (1:1000) diluted in 5\% normal goat serum in PBST for 30 minutes. After incubation the slides were exposed 30 minutes to horseradish peroxidase coupled vectastain $A B C$ reagent (vector laboratories, Burlingame, CA, USA), which binds to the biotinylated secondary antibodies. The immunostaining was revealed by a short incubation of 5 minutes to 3,3-diaminobenzidine tetrahydrochloride (DAB). The reaction was stopped by washing the slides with demineralized water followed by a counterstaining with haematoxylin.

\section{Histological Examination}

Photos were taken using a Leica Leitz DMRB fluorescence microscope (Leica Microsystems, Eindhoven, The Netherlands). Using a 20x objective, photos were taken from five high power fields with a size of $0.38 \mathrm{~mm}^{2}$ of the placental sections for each study group. Thus a tissue area of $1.9 \mathrm{~mm}^{2}$ was analysed per placenta. The number of villi, true knots and syncytial knotting and shed STBMs per high power field at 20x were counted using these pictures. The included villi had to be completely located within the $20 x$ high power fields.

\section{Results}

The number of villi and the frequency of true knots and syncytial knotting were analysed in 106 placentas. Syncytial nuclear aggregates (SNAs) include true knots, syncytial knotting and STBMs. They are analysed to conclude if true knots and syncytial knotting can be markers for dysregulated placental maturity. The results are displayed as the sum of $1.9 \mathrm{~mm}^{2}$ analysed placental tissue. 


\section{True Knots}

Pre-eclamptic placentas show significantly higher numbers of true knots (8.1 per $1.9 \mathrm{~mm}^{2}$ ) compared to the other placentas. A trend towards decreased true knots in placentas complicated by chorioamnionitis $\left(1.6\right.$ per $1.9 \mathrm{~mm}^{2}$ ) compared to the idiopathic preterm group ( 3.1 per $1.9 \mathrm{~mm}^{2}$ ) is identified. In addition, chorioamnionitis placentas have significantly less true knots compared to pre-eclamptic placentas ( $p$-value <0.0001).
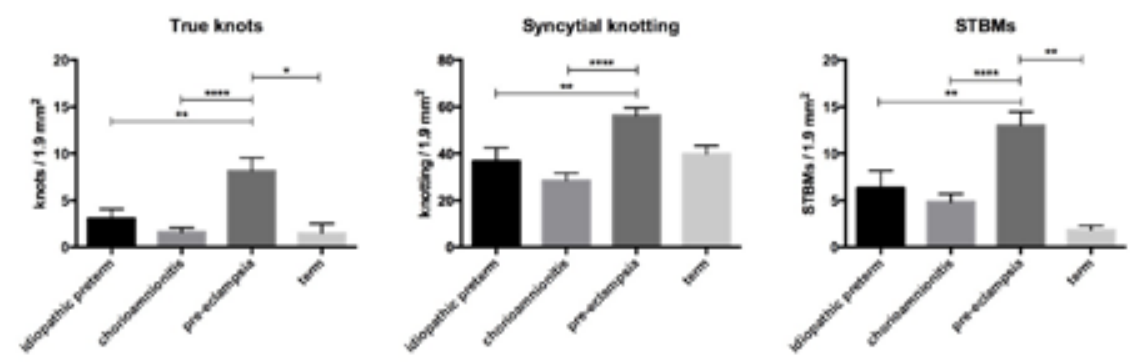

Figure 2. Number of counted true knots, syncytial knotting and STBMs. Data is given as the sum of five high power fields per analysed placenta with indicated SEM. True knots which show degenerative, apoptotic nuclei are significantly more present in pre-eclamptic placentas compared to the idiopathic preterm control group and term control group. Syncytial knotting is also significantly increased in pre-eclamptic placentas compared to idiopathic preterm control group and placentas complicated by chorioamnionitis. Number of STBMs are significantly increased in placentas complicated by pre-eclampsia compared to idiopathic preterm and term control and placentas complicated by chorioamnionitis. $\left({ }^{*} \mathrm{P} \leq 0.05 ;{ }^{* *} \mathrm{P} \leq 0.01 ;{ }^{* * *} \mathrm{P} \leq 0.001\right.$; $\left.{ }^{* * * *} \mathrm{P} \leq 0.0001\right)$

\section{Syncytial Knotting}

The number of observed syncytial knotting is significantly increased in pre-eclamptic placentas compared to idiopathic preterm ( $p$-value 0.0023) and chorioamnionitis placentas ( $p$-value <0.0001) (Figure 2). Numbers of syncytial knotting in term control placentas (39.83 per $1.9 \mathrm{~mm}^{2}$ ) are comparable with idiopathic preterm control placentas (36.71 per $1.9 \mathrm{~mm}^{2}$ ). Placentas complicated by chorioamnionitis show a trend towards decreased syncytial knotting (28.51 per $1.9 \mathrm{~mm}^{2}$ ) compared to all other placentas in conjunction with decreased number of true knots.

\section{STBMs}

Pre-eclamptic placentas have significantly higher numbers of STBMs in this study (Figure 2). They show on average 13 STBMs per placenta. Chorioamnionitis placentas have a trend 
towards decreased STBMs frequency (4.9 per $1.9 \mathrm{~mm}^{2}$ ) compared with idioapathic preterm placentas (6.3 per $1.9 \mathrm{~mm}^{2}$ ) and a significant decrease compared with pre-eclamptic placentas ( $p$-value <0.0001). Term placentas display the least STBMs of all analysed placental sections (1.8 per $\left.1.9 \mathrm{~mm}^{2}\right)$.

\section{Discussion}

The purpose of this study was to quantify true syncytial knots, syncytial knotting, STBMs in placentas derived from pregnancy pathologies. This was done by histological examination of 24 placentas obtained from idiopathic preterm labour, 41 placentas complicated by chorioamnionitis and 35 pre-eclamptic placentas. In addition, 6 placentas from uncomplicated term delivery were analysed to set a reference value for villous maturation.

\section{Pre-eclampsia}

In placentas complicated by pre-eclampsia the number of counted true syncytial knots, syncytial knotting and STBMs is significantly increased compared to our idiopathic preterm control group. Increased true syncytial knots are also seen in other previously published data $(2,17)$ demonstrating that our quantification method is consistent. The overall increase of syncytial knotting and increased number of smaller villi conveys the overall impression that pre-eclamptic placentas show more villous branching than other placentas of same gestational age. The histologic sections convey the impression that villi are more adjoining and branched probably to increase the area of diffusion. Also increased apoptotic shedding of syncytiotrophoblast cells is visible as an adaptation to uteroplacental malperfusion. Since pre-eclampsia is responsible for a higher placental maturation, the increased frequencies of true syncytial knots and syncytial knotting are relevant markers for villous maturation in pre-eclampsia. This enhanced maturation is possibly caused by placental hypoxia due to the aetiology of pre-eclampsia. Increased villous trophoblast turnover and formation of terminal villi may be a compensation of the placenta to try to assure foetus oxygen supply. Normally the trophoblast has many functions in foetal metabolism and changes of trophoblastic structure are necessary to nourish the developing foetus. In the third trimester the chorionic villous tree needs to differentiate to increase placental exchange to compensate for the reduced placental expansion rate. In pre-eclampsia it is necessary that the placenta adapts earlier to lower blood supply. 


\section{Chorioamnionitis}

The villous maturation in placentas complicated by chorioamnionitis is not well understood yet. This study showed reduced numbers of true syncytial knots and syncytial knotting in placentas with histologic evidence of intra-amniotic infection compared to pre-eclampsia and idiopathic preterm group. This could be interpreted as a sign for decreased placental villous maturation. A recently published study confirmed these results stating that the frequency of placental villous maturation is significantly higher in an idiopathic preterm group than in placentas with acute chorioamnionitis (18). This relation was independently of infection characteristics of the analysed cases. Only 33\% of all placentas complicated by funisitis, acute chorionitis and acute amnionitis showed placental villous maturation. From the slightly reduced syncytial nuclear aggregates in chorioamnionitis it can be assumed that other pathological mechanisms play a role during this infection. Our data suggest that there is no placental insufficiency in chorioamnionitis, otherwise the frequency of syncytial nuclear aggregates is assumed to be higher due to adaptations. Also the placental weight does not vary from idiopathic preterm group indicating that the placental volume is sufficient to provide the foetus with enough oxygen. It can be concluded that frequencies of true syncytial knots and syncytial knotting, in contrast to pre-eclampsia, are not adequate markers to characterize placental cases of chorioamnionitis.

\section{Conclusion}

We have quantified the number of true knots and syncytial knotting within villous tissue and described an increase in pre-eclamptic placentas. This increase indicates that the ratio of trophoblast turnover is increased in pre-eclampsia. These parameters can be used as markers for villous maturation in pre-eclampsia in our scoring system. In placentas complicated by chorioamnionitis the frequency of true knots, STBMs and syncytial knotting is only slightly decreased compared to the idiopathic preterm control group. Therefore, increased trophoblast turnover is not observed in chorioamnionitis and syncytial knots, STBMs and syncytial knotting cannot be used as markers for judging villous maturation in chorioamnionitis. Our second control group consisting of term placentas was too low in numbers to provide convincing results. From existing data it can be concluded that term placentas show the highest frequencies of true knots compared to preterm placentas due to an increased necessity to nourish the growing foetus with limited placental volume. 


\section{Role of the student}

Madita Reimer was an undergraduate student working under the supervision of Ulrike von Rango when the research in this report was performed. The topic was proposed by the supervisor. The histological examination, data analysis and interpretation were done by the student. The $\mathrm{CD}_{31}$ stainings of three preterm groups were done before by other project members and staining of the term group was done by the student.

\section{Acknowledgments}

First I wish to express my sincerest thanks to my supervisor, Ulrike von Rango, for her encouragement and support. Furthermore, in the laboratory I have been aided by Paul van Dijk who teaches me a lot about immunohistochemistry. I also want to thank Philippe Vangrieken for his daily support to get the research project started in the beginning of my internship. I am also grateful to all of the Department of Anatomy and Embryology for their kindness and including us interns in their team. Finally I would also like to thank my fellow students and my family for supporting me throughout my studies at Maastricht University.

\section{References}

1. Singh V. Textbook of Clinical Embryology: Elsevier Health Sciences APAC; 2014.

2. Benirschke K, Kaufmann P, Baergen RN. Pathology of the Human Placenta, 5th Edition: Springer; 2006.

3. Carlson BM. Human Embryology and Developmental Biology. 5 ed: Elsevier Health Sciences; 2012.

4. Fogarty NM, Mayhew TM, Ferguson-Smith AC, Burton GJ. A quantitative analysis of transcriptionally active syncytiotrophoblast nuclei across human gestation. J Anat. 2011;219(5):601-10.

5. Huppertz B. Placental villous trophoblast: the altered balance between proliferation and apoptosis triggers pre-eclampsia. Journal für Reproduktionsmedizin und Endokrinologie-Journal of Reproductive Medicine and Endocrinology. 2006;3(2):103-8.

6. Fox H. The significance of villous syncytial knots in the human placenta. J Obstet Gynaecol Br Commonw. $1965 ; 72: 347-55$

7. Jones CJ, Fox H. Syncytial knots and intervillous bridges in the human placenta: an ultrastructural study. Journal of Anatomy. 1977;124(Pt 2):275-86.

8. Kubli F, Budliger H. Contribution to the morphology of placental insufficiency. Geburtshilfe Frauenheilkd. $1963 ; 23: 37-43$.

9. Tenney B, Parker F. The placenta in toxemia of pregnancy. Am J Obstet Gynecol. 1940;39:1000-5

10. Calvert SJ, Jones CJP, Sibley CP, Aplin JD, Heazell AEP. Analysis of syncytial nuclear aggregates in preeclampsia shows increased sectioning artefacts and decreased inter-villous bridges compared to healthy placentas. Placenta. 2013;34(12):1251-4. 
11. Naeye RL. Functionally important disorders of the placenta, umbilical cord, and fetal membranes. Human pathology. 1987;18(7):680-91.

12. Grether JK, Eaton A, Redline R, Bendon R, Benirschke K, Nelson K. Reliability of placental histology using archived specimens. Paediatr Perinat Epidemiol. 1999;13(4):489-95.

13. Stark MW, Clark L, Craver RD. Histologic Differences in Placentas of Preeclamptic/Eclamptic Gestations by Birthweight, Placental Weight, and Time of Onset. Pediatric and Developmental Pathology. 2014;17(3):1819.

14. Ruiz-Quinonez G, Reza-Lopez SA, Chavez-Corral DV, Sanchez-Ramirez B, Leal-Berumen I, Levario-Carrillo M. Placental maturity, hypertensive disorders of pregnancy and birth weight. Hypertens Pregnancy. 2014;33(2):132-44.

15. Rayburn W, Sander C, Barr M, Jr., Rygiel R. The stillborn fetus: placental histologic examination in determining a cause. Obstet Gynecol. 1985;65(5):637-41.

16. Huppertz B, Kaufmann P, Kingdom J. Trophoblast turnover in health and disease. Fetal and Maternal Medicine Review. 2002;13(02):103-18.

17. Heazell AEP, Moll SJ, Jones CJP, Baker PN, Crocker IP. Formation of Syncytial Knots is Increased by Hyperoxia, Hypoxia and Reactive Oxygen Species. Placenta. 2007;28, Supplement(0):S33-S40.

18. Morgan TK, Tolosa JE, Mele L, Wapner RJ, Spong CY, Sorokin Y, et al. Placental villous hypermaturation is associated with idiopathic preterm birth. The Journal of Maternal-Fetal \& Neonatal Medicine. 2012;26(7):647-53. 